\title{
On Validity of Paleomagnetic Data and Transitional Regime of Geomagnetic Field
}

\author{
G.N. Petrova \\ Geophysical Institute of the Academy of Sciences of the USSR \\ B. Gruzinskaya, 10, Moscow, USSR.
}

A great number of paleomagnetic data, accumulated by present time puts on the agenda the question of these data interpretation. The attempts of such interpretation, taken again and again by different investigators, give rise to hot discussions, but cause distrust of some geophysists to paleomagnetic method as a whole. It is enough to remember the report of professor Thellier in Berkeley.

Simultaneously with it, and I should say, independently on it, the number of paleomagnetologists feel an increasing dissatisfaction by that validity, which paleomagnetic investigations assure at present. Not speaking about the results, obtained some years ago, when as a rule paleomagnetic data have been published without any proof of their validity, but even in the last papers authors often limit themselves only by very primitive determinations of initial magnetization. It is necessary to add, that in principle we can't distinguish CRM from TRM and that our knowledge how the different types of residual magnetizations under the effect of strains leaves much to be desired. As things now stand it is difficult to guarantee for correctness of geophysical generalizations, which are carried out on the basis of paleomagnetic data.

Though this phrase should not be understood pessimistically. Already at present stage of paleomanetic investigations geophysics have obtained principally new data from paleomagnetism. So for example we can discuss about reality of such important for our imaginations fact about the internal structure of the Earth, as geomagnetic field inversion. I venture to refer to Hramov works-his paper had been read on the Conference in Berkeley. Having compared the numerous results of different authors, which have worked on the Russian platform and in Siberia, the regions, removed from each other by some thousands of kilometers and isolated absolutely geologically, Hramov have obtained a full agreement in the alternation of straight and reverse zones of these regions (Fig. 1).

The same good coincidence is observed for Permo-Triassic of the European part of the USSR and North America (Fig. 2).

Planetary propagation of zones of normal and reverse magnetization is the best evidence of inversions reality.

Hramov showed, that in different epoches a different regime of geomagnetic field had taken place: so, for instance, in Ordovician and Devonian often replacement of directions had taken place, in upper Perm and Triass the field of one sign had existed during more long time, but both directions of field are of equal probability, while in Quaternary the 


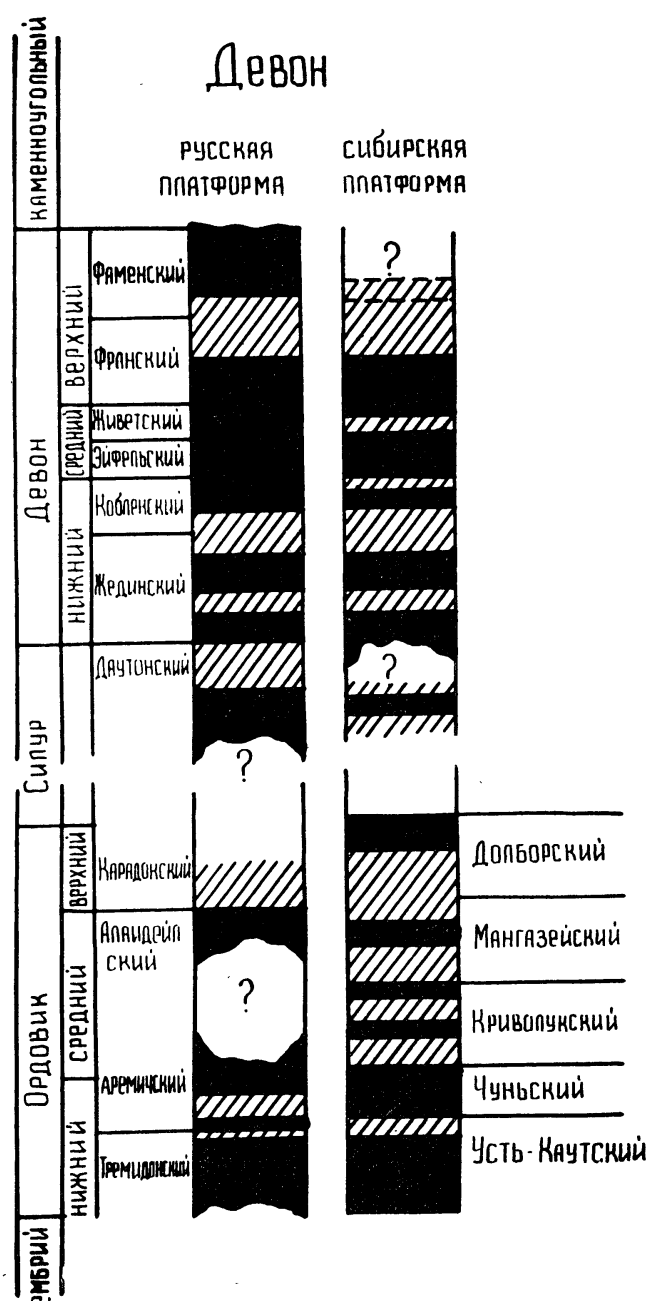

Fig. 1 Comparison of paleomagnetic sections of the Russian and Siberian platforms for Ordovician-Devonian.

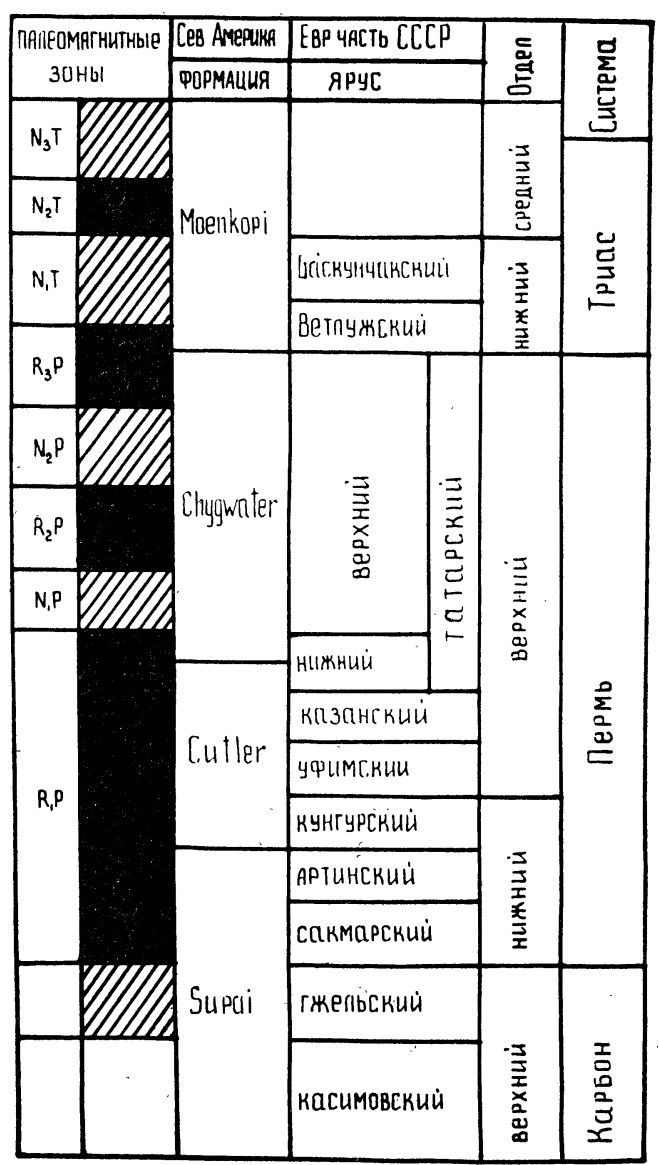

Fig. 2 Comparison of paleomagnetic sections of the European part of the USSR and North America for Carbonferous-Triassic periods.

normal magnetization prevails, but in Carboniferous period and low Perm the reverse one. So long as this result, obtained by numerous and studied in detail paleomagnetic sections in the European part of the USSR and Siberia, is proved by the data of two continents, it is possible to believe it, though little places of sections don't agree by the sign. The fact is that at studying concrete sections authors not always can, but some times even don't try to prove the geophysical origin of reverse magnetization. Because of it the World Paleomagnetic Summary includes unreliable, and sometimes incorrect data, confusing general picture. Both such proofs are not only possible but from my point of view are quite obligatory. I can refer to 4 works of the Soviet authors, where by the combination of laboratory investigations of stability and petrographycal, chemical and structural analyses it have been a success to prove singlemeaningly the reality of inversions in concrete case. This is the work of Bolshakov and Solodovnikov on Pliocene of Armenia the work of Mandrusova 

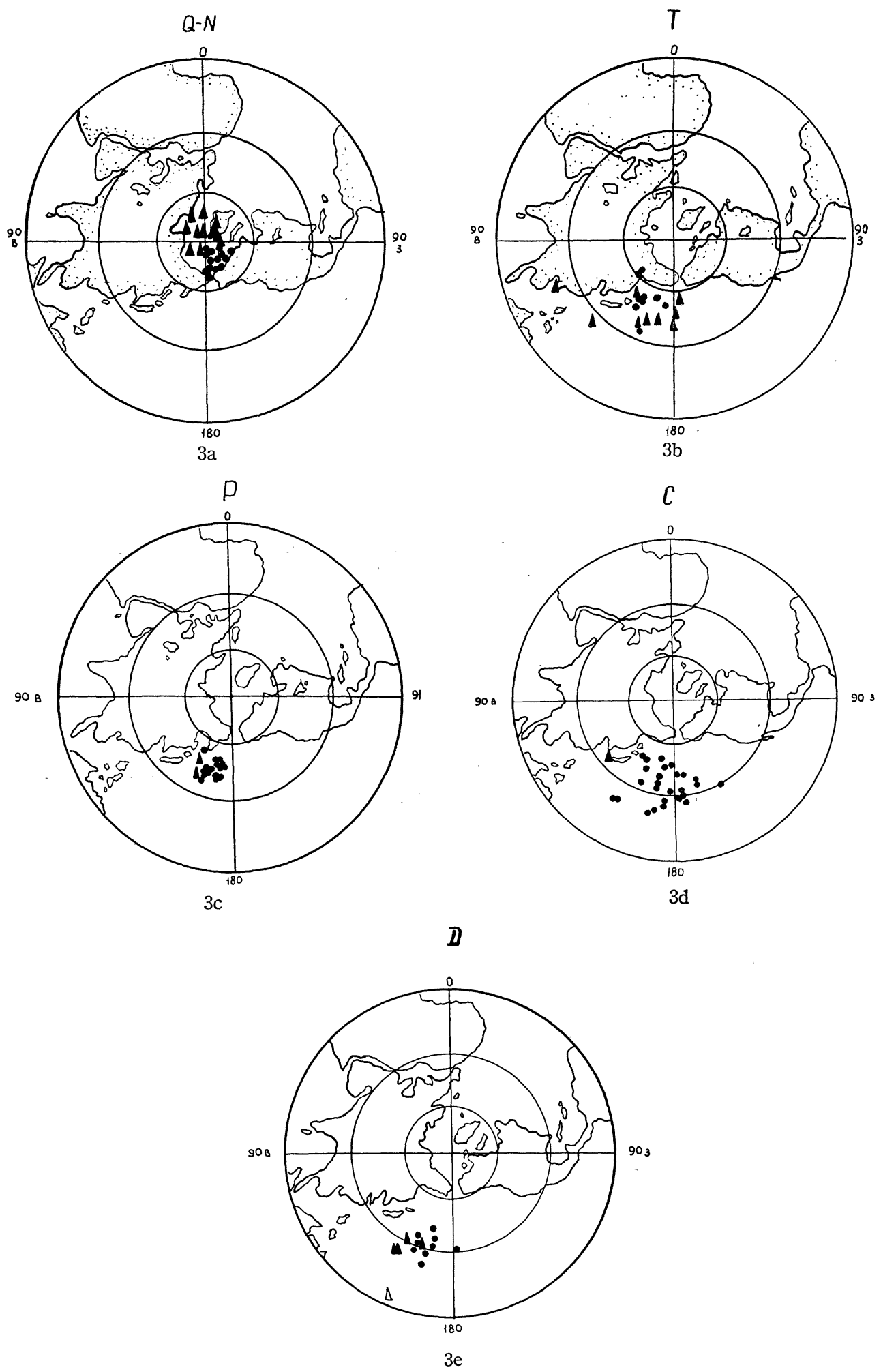

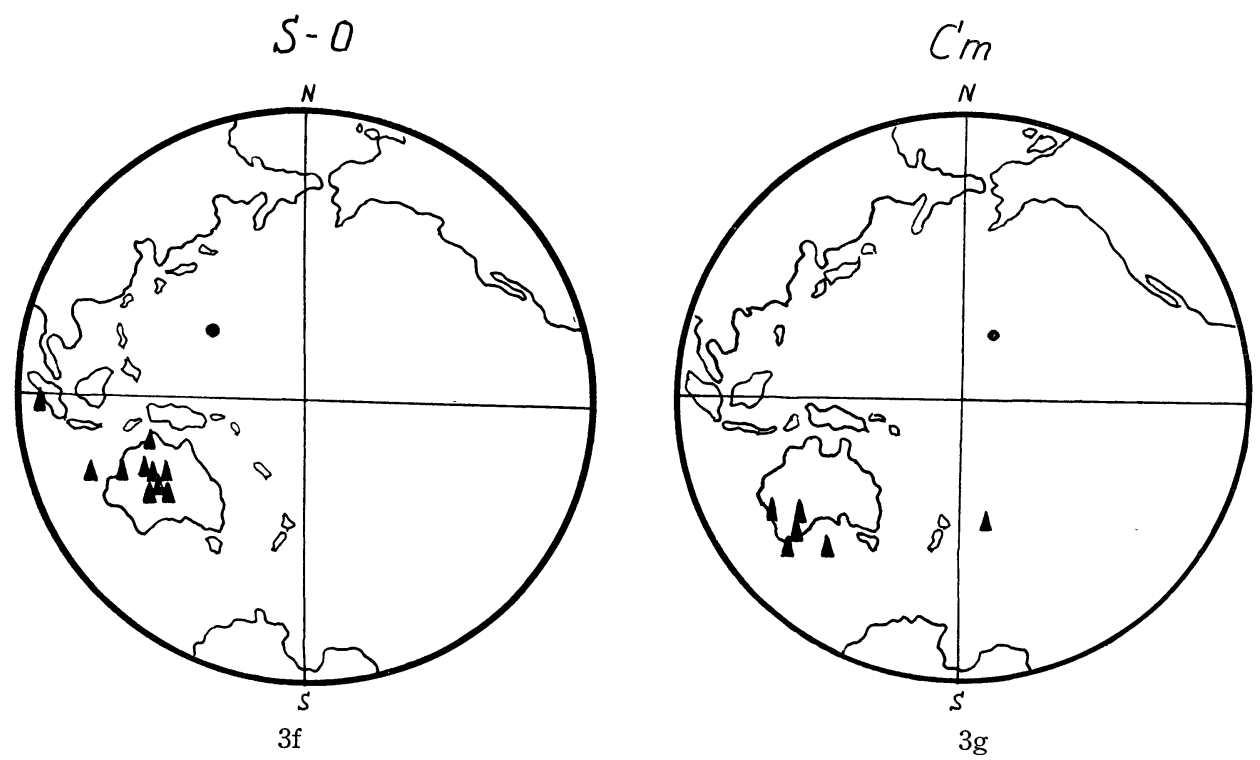

Fig. 3 Position of virtual poles, determined by the rocks of European part of the USSR and Siberian platform in Neogene (3a), Triassic (3b), Permian (3c), Carboniferous (3d), Devonian (3e), Silurian-Ordovician (3f), Cambrian (3g).

and my on Pliocene of Far East, Vlasov work on Devonian of Krasnojarsky land and our work on Pliocene of Turkmenia and Azerbaijan, about which I shall speak in the second part of my paper.

Necessity to have paleomagnetic data of high validity especially sharply is observed at attempt to explain the difference of paleomagnetic data on different continents. Particularly such difference is observed and by the data of the Siberian and Russian platforms (Fig. 3).

After evaluation of having results, Hramov comes to the conclusion, that on present level of our knowledges about $J_{n}$ formation and its variation with time, we can not explain the distribution of paleomagnetic poles, without using hypothesis, that Asia and East Europe have changed their position relatively each other, that, in particular, the turn of one of them relatively other by angle of $40^{\circ}$ order.

It is necessary to note, that the data according to Siberia and to the Russian platform in the first approximation are in satisfactory coincidence with paleoclimatic data, that makes Hramov supposition more well-founded. However, when we have made an attempt to select the data, quite reliable both from geological and magnetic points of view, which could be the "base" and the on the basis of which it should be possible to make an idea about character of this difference we could hardly select 2-3 points for every epoch.

On the other hand, the great number of concrete collections, with which we have met in our laboratory,- - but we in the Earth Physics Institue and the Moscow University engage in for not so much paleomagnetic investigations as just for the problems of validity, makes me to prove, that at the result of detailed investigations our idea about NRM origin of investigating collection and about its initial direction sometimes is strongly changing. I am not afraid to confess, that not always in general we are a success to have the answer 
these questions.

It seems to me, that now we are mostly interested in obtaining reliable paleomagnetic data, where the regularity of determination of ancient local field may be proved.

In our investigations, particularly, in that one, to which the second part of my paper is devoted, we use the next methods for ascertaining the results validity:

Working with igneous rocks by successive heating method with an obligatory analysis of change in the time of heating of magnetization direction and with the determination of coefficient " $K$ " (of ancient field relation to laboratory one). The analysis of $J_{r s}\left(t^{\circ}\right)$ curve, showing Curie points, phase transitions, oxidation, dehydratization and variations of this curve at repeated heating.

At working with any rocks we use the method of stability characteristics comparison, i.e. comparison of mutual position of curves $J_{n}(\widetilde{H}), J_{r i}(\widetilde{H}), J_{r}(\widetilde{H})$, and sometimes $J_{r t}(\widetilde{H})$ and laboratory field determination, at which we obtain $J_{r i}=J_{n}$.

The analysis of $J_{n}(\widetilde{H})$ curve in the fields to 500 Oe with particular attention to $J_{n}$ direction change in demagnetization process. Determination of $H^{1}$ and viscosity coefficients.

Low temperature investigations

Repricipitation.

Petrographical analysis of ferromagnetic fraction composition and primariness of ferromagnetic grains. By the way the standard petrographical descriptions are not suitable for this aim.

Roentgenstructural analysis with the aim of accurate definition of composition by a constant lattice.

Sometimes all this is not enough and it is necessary to use special chemical analyses, electron microscope and so on.

The complex of such investigations makes it possible in some cases to find out singlemeaningly NRM origin, i.e. to prove the results validity, in other cases to give more or less confident supposition. Even in that cases, when we are not a success to prove correctness of results, we obtain the material, which, possibly, in consequence gives the way to a singlemeaning interpretation, when our knowledges about formation and destruction of magnetization will increase.

I should like now to turn to summary of the work results, which as it seems to us represent any interest for the theory of the origin of the main geomagnetic field, and, consequently for the study of processes in the Earth core.

We have investigated the same inversion of magnetic field in Pliocene, and just $N_{3}-R_{2}$ on the samples of Azerbaijan and the West Turkmenia.

In Turkmenia the section is constructed of red clay, interstratificated by sand. The thickness of section is $40 \mathrm{~m}$, inclination agnle $-15-20^{\circ}$, azimuth $-315-325^{\circ}$. In Azerbaijan the thickness of section is $20 \mathrm{~m}$. Bedding elements are 50 and 290 . The section is constructed of grey-brown and grey clays and grey aleurolite. The samples by dimension, which is $2,5 \times$ $2,5 \times 2,5 \mathrm{~cm}$ have been selected with the interval of $5,0 \mathrm{~cm}$.

We consider, that only explanation of sign variation of magnetization in this case is the field inverstion, as: 
1. Stability in relation to a constant and alternate fields is the same of the samples of the normal, reverse and transitional zone.

2. The composition of ferromagnetic fraction of all three zones as to the data of petrographical and roentgenstructural analysis is the same. Magnetic parameters, such as $c, J_{r i}(H), J_{r s}, H_{c s}$ prove it.

3. In Azerbaijan, where the only ferromagnetic of clays carrying on magnetization is magnetite, the value of $J_{n}$ of normal and reverse zones is the same.

4. The rocks of Azerbaijan and Turkmenia essentially differ from each other by the composition of ferromagnetic fraction and by stability parameters, though, the variation of magnetization sign of them is observed simultaneously (Surahovskaja suite of Azerbaijan is geological analogy of Turkmenian suite of the red colour clays).

5. In transitional zone a sharp variation of direction is observed in the limits of one layer of clay, while in a normal and reverse zone the directions of $J_{n}$ coincide for layers, different by designation and by all their characteristics, but neighbouring by the position in the section.

6. The experiments, connected with repricipitation show the same ratio between $J_{n}$ and $J$ precipit for the samples of normal and reverse zones.

Relative change of the ancient field value have been estimated by laboratory field value, in which $J_{r i}$ have been equal to $J_{n}$.

We considered, that determine correctly the initial $J_{n}$, if at demagnetization in the fields of $150-500$ the direction of magnetization was not changed and if curve $J_{n}(\widetilde{H})$ was placed above curve $J_{r i}\left(\widetilde{H}_{i}\right)$. By additional criterion is coincidence of directions $J_{n}$ of neighbouring lithological differences, or - at a sharp variation of directions, even inside one layer-equality

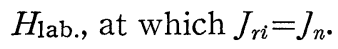

$J_{n}$ of only that samples are drawn on stereogramms, which satisfied these demands. (Fig. 4).

The detailed investigation of direction and value of magnetization of the rocks of Azerbaijanian and Turkmenian sections make it possible to compell the next scheme of transitional regime of geomagnetic field.

I. The sharp and quick change of field direction by azimuth (variation of declination). Simultaneously the decrease of the field intensity is observed (Fig. 4, 5, 6).

II. The increase of variations amplitude. Variations are of unregulating character. At the end of this stage a little increase of the field intensity ensures.

III. Variations assume a regulating character of "swing" i.e. form long narrow loops with almost invariable direction of long axis (Fig. 7).

The field intensity here sharply decreases to $0,3-0,5$ of initial value. In the process of these variations the inclination little by little changes for negative one.

IVa. Variations become irregular, though their amplitude is still great. New increase of the field intensity is observed.

IVb. Variations are damping gradually. The field declination is close to the declination in a negative stationary position, inclination is negative, but differs from stationary meaning. The field intensity is slightly decreasing. Sand interlayers and pinching out of clays in Turkmenian section don't allow to compare step by step the development of transitional 


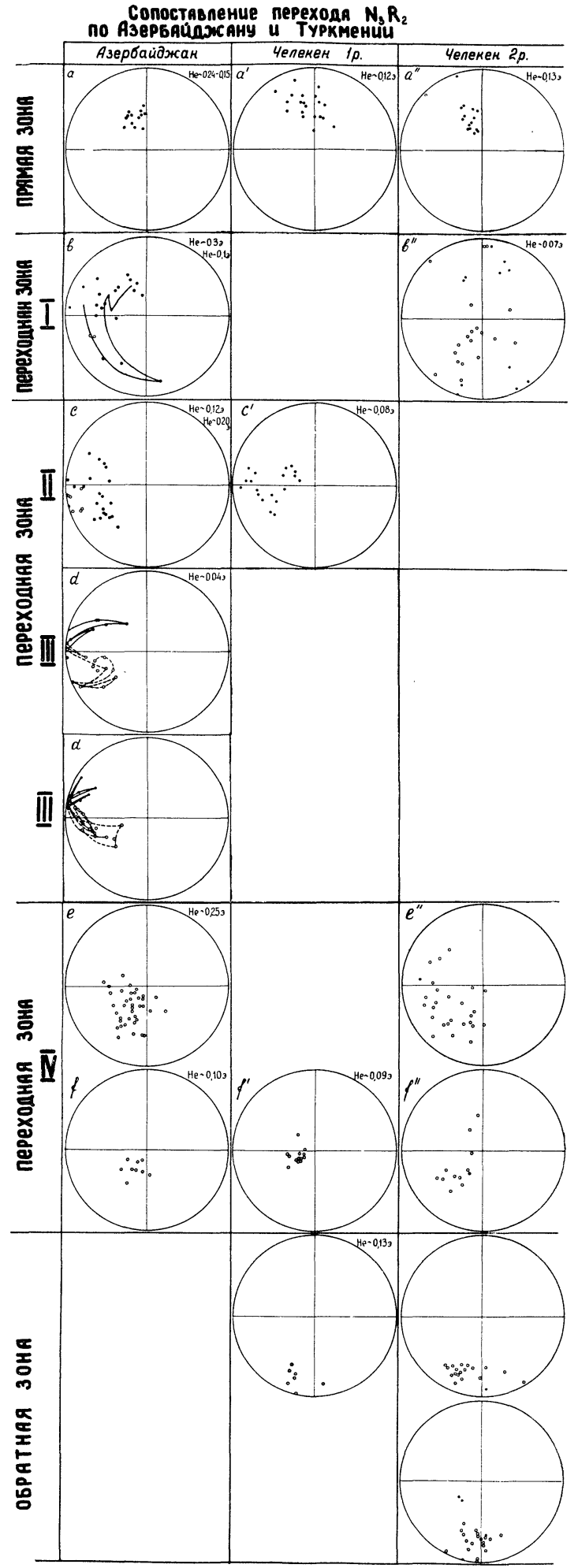

Fig. 4 Stereograms of transitional zone $N_{3}-R_{2}$ of Azerbaijan and Cheleken sections (Pliocene). 


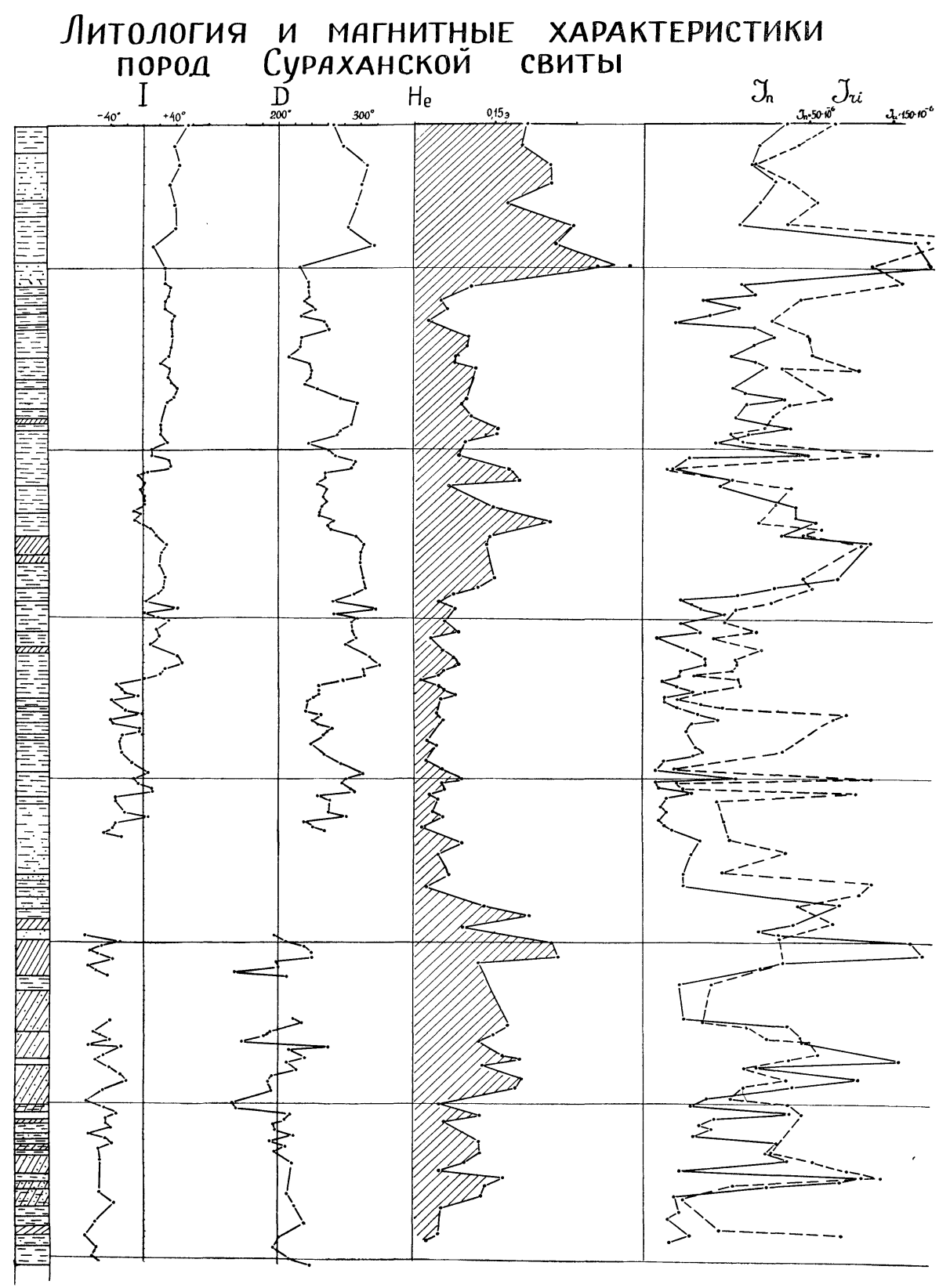

Fig. 5 Lithology and magnetic characteristics of Surakanskaja suite rocks.

regime in two investigated regions. However according to the position of the average points of transition there is a ground to consider, that an average direction of the axis displacement in these two regions had been one and the same. The variation of the field intensity, which is by the way very well revealed and according to the reprecipitation data, is also observed in both regions.

It is possible to evaluate approximately the time of transition. According to geology 


\section{Cxema nepexoda $N_{3} R_{2}$}

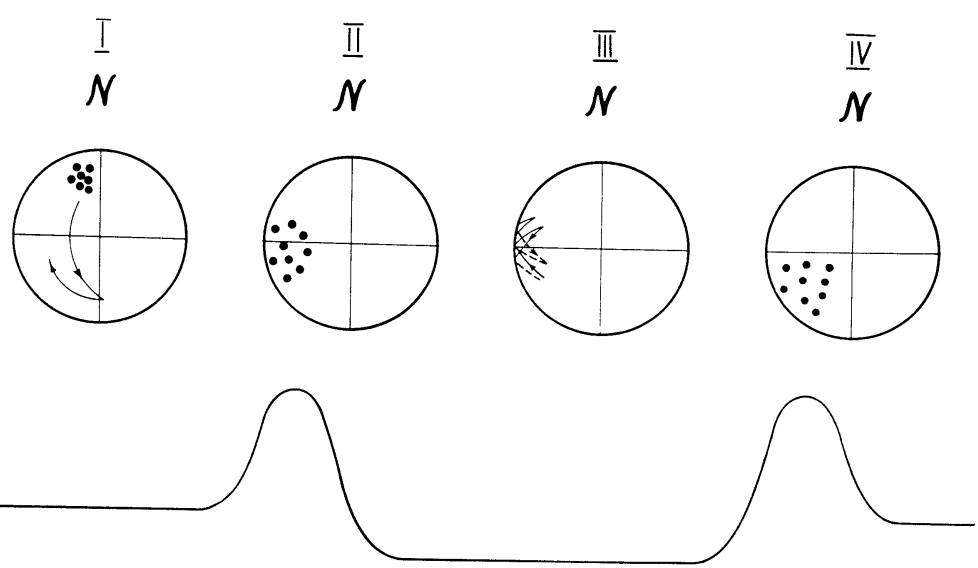

Fig. 6 Scheme of transitional regime of geomagnetic field $\left(N_{3}-R_{2}\right.$, Pliocene).

data sedimentation in Azerbaijan have occured very uniformly. Surakanskaja suite, a general thickness of which is $800 \mathrm{~m}$., have been accumulated for $10^{6}$ years (rather less). Thus, the transition, occupying $16 \mathrm{~m}$, is lasting for 16-20 thousands of years. Every cycle of "swing" occupying $1 / 2$ of a meter, is lasting $500-600$ years.

The periods of the same order find both secular variations of magnetic moment and the directions of geomagnetic field.

In our disposal we had the collections of some transitions else. Unfortunately, no one of them couldn't secure enough full picture of the field variation at inversion, as Azerbaijan collection, but the intensity decrease of the field while transition is observed in all the cases. 


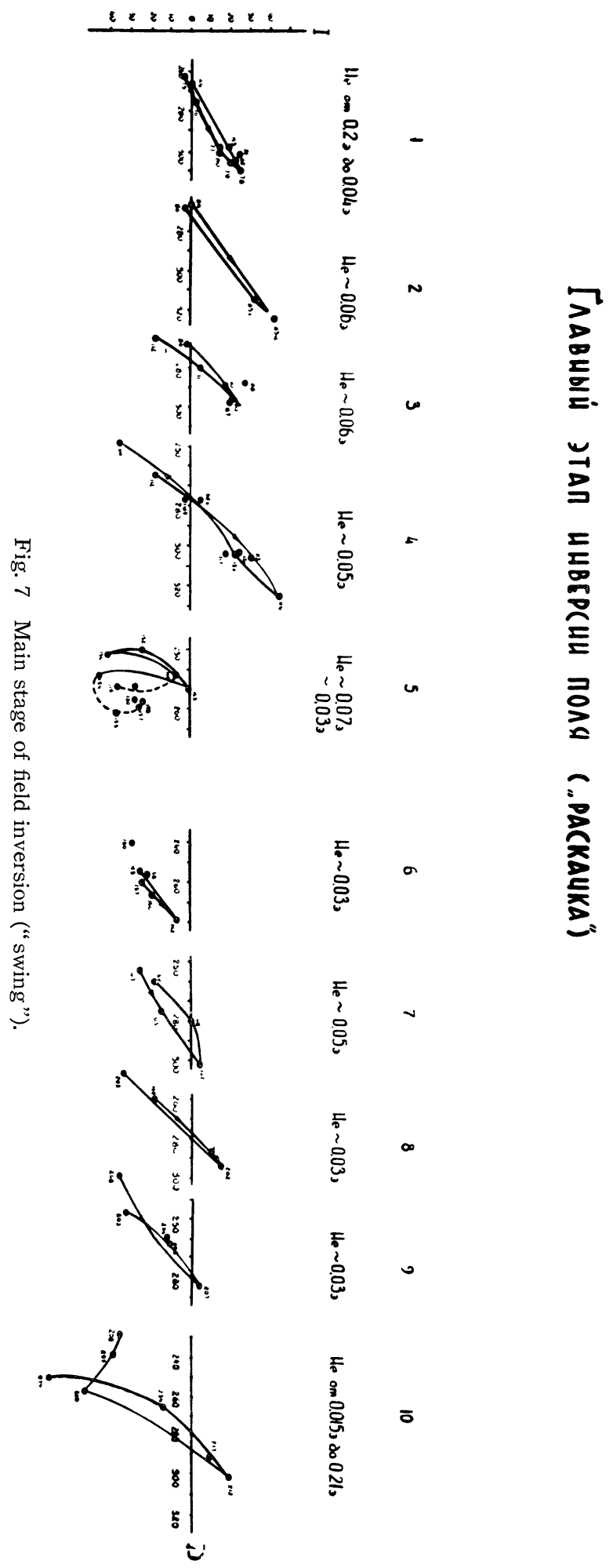

